\title{
High-order Harmonic Field Retrieval in Ethylene
}

\author{
Varun Makhija ${ }^{1}$, Jan Tross ${ }^{2}$, Vinod Kumarappan ${ }^{2}$, Michael Spanner ${ }^{1}$, Caterina Vozzi $^{3}$, Car- \\ los Trallero ${ }^{4}$, and Salvatore Stagira ${ }^{3, *}$ \\ ${ }^{1}$ Department of Physics, University of Ottawa, 598 King Edward, Ottawa, Ontario K1N 6N5, Canada \\ ${ }^{2}$ Department of Physics, Kansas State University, 116 Cardwell Hall 1228 N. 17th St., Manhattan, KS \\ 66506-2601, USA \\ ${ }^{3}$ Dipartimento di Fisica, Politecnico di Milano \& IFN-CNR, piazza L. da Vinci 32, 20133 Milano, Italy \\ ${ }^{4}$ Department of Physics, University of Connecticut, 2152 Hillside Rd, U-3046 Storrs, CT 06269-3046, \\ USA
}

\begin{abstract}
The XUV field emitted by impulsively aligned ethylene molecules during high-order harmonic generation is retrieved as a function of molecular orientation. The results can be ascribed to multielectron contributions to the harmonic emission.
\end{abstract}

\section{Introduction}

High-order harmonic generation (HHG) is a formidable tool for investigating the intimate nature of atoms and molecules, since the properties of the harmonic radiation strongly depend on the outermost electronic structure of the target species. Spectroscopic applications of HHG in molecules require however to retrieve the XUV harmonic field as a function of the molecular orientation through molecular alignment techniques [1].This goal is easily achieved in linear molecules, but its extension to general cases is not trivial. In this work we show that this issue can be overcome by exploiting rotational coherence spectroscopy (RCS) [2, 3]. As a benchmark case we focused our investigation on ethylene, that shows a planar geometry.

\section{Experimental results and XUV field retrieval}

We generated high-order harmonics in a jet of $\mathrm{C}_{2} \mathrm{H}_{4}$ molecules at a rotational temperature of $36 \mathrm{~K}$. Impulsive molecular alignment was induced by $800-\mathrm{nm}, 130$-fs pulses with a peak intensity of $13.6 \mathrm{TW} / \mathrm{cm}^{2}$ and harmonic generation was driven by a delayed $800-\mathrm{nm}, 30$ fs laser pulses with a peak intensity of about $170 \mathrm{TW} / \mathrm{cm}^{2}$. The two pulses were linearly polarized along the same direction, indicated hereafter as $Z$ axis. Harmonic spectra were recorded as a function of the delay $\tau$ between the pulses; the intensity of each harmonic was then obtained by spectral integration of the harmonic peak and normalization with respect to the intensity measured at negative delays. The left panel in Fig. 1 shows the results; for the sake of clarity, the figure shows an interpolation of the harmonic signal along the spectral dimension. Partial rotational revivals are clearly visible along the delay scan; in addition a modulation of the harmonic intensity as a function of the harmonic order is also observed.

\footnotetext{
*e-mail: salvatore.stagira@polimi.it
} 

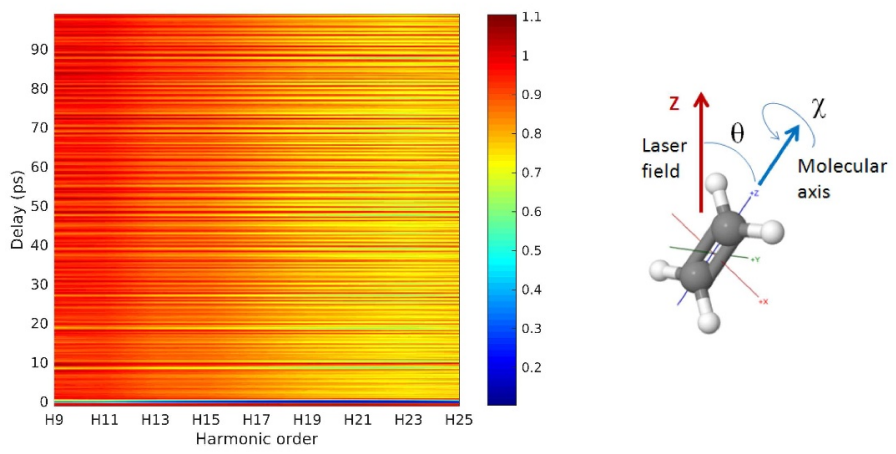

Figure 1. Left: normalized HHG spectra recorded in impulsively aligned ethylene molecules as a function of the delay $\tau$ between aligning and driving laser pulses. Right: description of the ethylene molecular frame.

In order to retrive the XUV harmonic field as a function of the molecular orientation, we calculated the temporal evolution of the excited rotational wavepacket induced in the ethylene molecules and we modeled the macroscopic harmonic intensity according to the relation:

$$
I(\omega, \tau) \propto\left|\int \rho(\theta, \chi, \tau) E_{Z}(\omega, \theta, \chi) d \theta d \chi\right|^{2}
$$

where $\rho(\theta, \chi, \tau)$ is the time-varying rotational distribution of ethylene molecules and

$$
E_{Z}(\omega, \theta, \chi)=\sum_{j k} C_{j k}(\omega) D_{0 k}^{j}(\theta, \chi)
$$

is the projection of the single-molecule XUV field on the $Z$ axis, $\theta$ and $\chi$ are the angles describing the orientation of the molecule according to the right panel in Fig. $1, C_{j k}$ are complex coefficient depending on the harmonic order and $D_{0 k}^{j}(\theta, \chi)$ are a subset of the Wigner D functions, forming in our case a complete basis set over which $E_{Z}$ is projected. Due to the experimental configuration, only the projection of the single-molecule XUV field along the $Z$ axis can be retrieved.

The retrieval was restricted to the coefficients $C_{00}, C_{20}, C_{22}, C_{40}$ and $C_{42}$ owing to the symmetry of the molecule that imply even values of the $j, k$ indexes with $C_{j(-k)}=C_{j k}$. For each harmonic order, the fitting procedure seeks for the global minimization of the difference between the experimental and the retrieved traces; this minimization occurs in two steps, exploiting a genetic algorithm followed by a deterministic one.

Left panel of Fig. 2 shows the excellent agreement between the experimental and the retrieved traces for harmonics $13^{\text {th }}$ and $23^{\text {rd }}$; similar results were obtained for all harmonic orders up to the cutoff. The retrieved complex coefficients were used in Eq. (2) for calculating the singlemolecule XUV field $E_{Z}(\omega, \theta, \chi)$ both in amplitude and phase. XUV amplitude and phase for harmonics $13^{\text {th }}$ and $23^{\text {rd }}$ are shown respectively in central and right panels of Fig. 2. The field structure in the molecular frame clearly changes with harmonic order.

A preliminary model based on the Strong Field Aproximation (not shown) shows that the retrieved XUV field at lower harmonic orders is influenced by the contributions of several orbitals, demonstrating the multielectron nature of the harmonic emission. However this model fails to reproduce the shape of the retrieved field at high orders. This departure may 




Figure 2. Left panels: experimental (orange) and retrieved (blue) traces for harmonics $13^{\text {th }}$ (upper panel) and $23^{\text {rd }}$ (lower panel). Central and right panels: retrieved XUV field amplitude and phase for harmonics $13^{\text {th }}$ (upper panels) and $23^{\text {rd }}$ (lower panels).

be ascribed to several contributions such as the structure of the Dyson orbital describing the multielectron nature of the molecule, the electronic wavepacket dynamics in the ion, the dressing effect of the laser field as well as the colliding wavepacket distortion induced by the ion Coulomb field. Further theoretical investigations involving the multielectron response in HHG including field-dressing effects are under way.

\section{Conclusions}

We have demonstrated the feasibility of single-molecule harmonic field retrieval in asymmetric tops exploiting rotational coherence spectroscopy. The results evidence the multielectron nature of harmonic emission in ethylene; further theoretical investigations aiming at a deeper understanding of the outcomes are ongoing. The exploitation of RCS paves the way to the extension of HHG spectroscopy to complex molecules.

\section{References}

[1] J. Itatani, J. Levesque, D. Zeidler, H. Niikura, H. Pépin, J. C. Kieffer, P. B. Corkum \& D. M. Villeneuve, "Tomographic imaging of molecular orbitals", Nature 432, 867 (2004).

[2] V. Makhija, X. Ren, D. Gockel, A.-T. Le, V. Kumarappan, "Orientation Resolution through Rotational Coherence Spectroscopy”, arXiv:1611.06476v2 [physics.atom-ph].

[3] X. Wang, A.-T. Le, Z. Zhou, H. Wei, and C. D. Lin, "Theory of retrieving orientationresolved molecular information using time-domain rotational coherence spectroscopy", Phys. Rev. A 96, 023424 (2017). 